\title{
Electron beam fabrication and characterization of high-resolution magnetic force microscopy tips
}

\author{
M. Rührig, ${ }^{\text {a) }}$ S. Porthun, and J. C. Lodder \\ MESA Research Institute, ISTG, University of Twente, PO Box 217, 7500 AE Enschede, The Netherlands \\ S. McVitie, L. J. Heyderman, A. B. Johnston, and J. N. Chapman \\ Department of Physics and Astronomy, University of Glasgow, Glasgow G12 8QQ, United Kingdom
}

(Received 26 June 1995; accepted for publication 22 August 1995)

\begin{abstract}
The stray field, magnetic microstructure, and switching behavior of high-resolution electron beam fabricated thin film tips for magnetic force microscopy (MFM) are investigated with different imaging modes in a transmission electron microscope (TEM). As the tiny smooth carbon needles covered with a thermally evaporated magnetic thin film are transparent to the electron energies used in these TEMs it is possible to observe both the external stray field emanating from the tips as well as their internal domain structure. The experiments confirm the basic features of electron beam fabricated thin film tips concluded from various MFM observations using these tips. Only a weak but highly concentrated stray field is observed emanating from the immediate apex region of the tip, consistent with their capability for high resolution. It also supports the negligible perturbation of the magnetization sample due to the tip stray field observed in MFM experiments. Investigation of the magnetization distributions within the tips, as well as preliminary magnetizing experiments, confirm a preferred single domain state of the high aspect ratio tips. To exclude artefacts of the observation techniques both nonmagnetic tips and those supporting different magnetization states are used for comparison. (C) 1996 American Institute of Physics.[S0021-8979(95)04023-5]
\end{abstract}

\section{INTRODUCTION}

Magnetic force microscopy (MFM) has proved to be a useful characterization tool for magnetic recording materials (see for example Ref. 1 and references therein). Both their intrinsic domain structure and written bit patterns can be investigated with high-resolution and minimal sample preparation. But MFM is an indirect domain observation technique, mainly probing the stray field emanating from the surface and not the magnetization vector within the sample. Therefore a comparison of a micromagnetic model for the sample magnetization and the stray field derived therefrom with the measured MFM signals is usually required for a quantitative interpretation of the images. As in other scanning probe techniques, however, the formation of the MFM signal is not straightforward in most cases due to perturbations arising from tip artefacts, surface topography, or tipsample interactions which are in general difficult to separate. An especial problem is the usually unknown magnetic state of the tip and the fact that its magnetic behavior in the stray field emanating from the sample may lead to perturbations of the observed MFM signal and misinterpretation of the images. Therefore, detailed magnetic characterization of the tips used is required in order to allow a quantitative interpretation of MFM images. Various experiments carried out in this field in the past were mainly focused on the observation of the stray field emanating from the tips because of the low electron transparency of common tips. Either Lorentz microscopy $y^{2-4}$ or electron holography ${ }^{5}$ has been used. Consequently only indirect information with respect to the magnetization within MFM tips could be obtained and the effec-

${ }^{a)}$ Present address: Dept. of Physics and Astronomy, University of Glasgow, Glasgow G12 8QQ, United Kingdom. tive domain structure was only accessible by modelling the measured MFM signal. ${ }^{6}$ Due to their good electron transparency, electron beam fabricated tips offer the possibility to study the microstructure and the domain structure within the tip itself using appropriate imaging techniques in a transmission electron microscope. Thus direct access to the domain configuration is possible and the magnetic behavior of this type of tip can be studied in more detail.

\section{ELECTRON BEAM FABRICATION OF THIN FILM MFM TIPS}

We recently demonstrated that electron beam fabricated tips offer a lot of advantages if used in MFM. ${ }^{7}$ There the magnetic volume is formed by a high aspect ratio thin film element deposited on a tiny carbon contamination needle which is prepared in a standard scanning electron microscope (SEM). The preparation process is shown in Fig. 1 together with a SEM picture of a tip prepared on top of a bent tungsten wire cantilever. The needles can be grown on all types of cantilever and their position and orientation is easily controllable during the growing process. Whilst the diameter of the needles is more or less constant (in our case: about 200 $\mathrm{nm}$ ) mainly depending on the stability of the SEM used for the preparation, their length can be varied by adjusting the dwelling time of the focused electron beam. In addition the magnetic properties of the tips can be tailored over a wide range by using appropriate magnetic coating materials. If the needles are grown slightly off center, the shadowing effect can be used during the evaporation of the magnetic material to form magnetically isolated high aspect ratio tips. The very good reproducibility of the preparation process, the high shape anisotropy, the parallel side walls, the smooth carbon surface, and the rounded apex of the needles should lead to a 


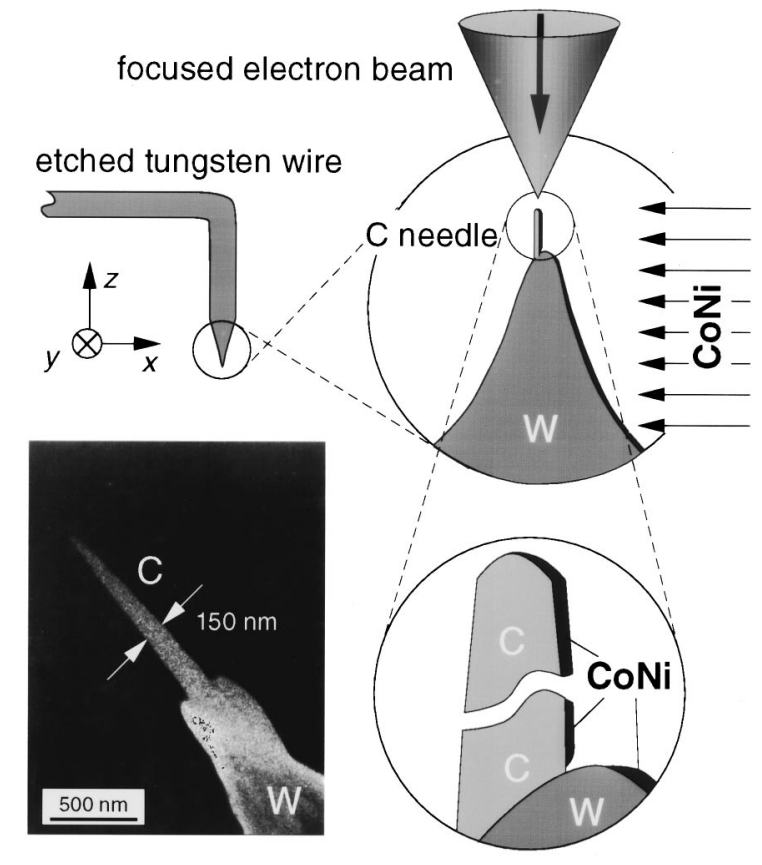

FIG. 1. Electron beam fabrication of MFM tips. On top of the cantilever a carbon contamination needle is grown and covered from the side with a magnetic layer $\left(\mathrm{Co}_{80} \mathrm{Ni}_{20}\right.$ alloy) to form an isolated thin film element.

thin film element tip with a well defined single domain magnetization state and therefore with an improved switching behavior. MFM experiments as well as transmission electron microscopy (TEM) studies of the tip stray field and the internal magnetization distribution are presented below as confirmation.

\section{MFM OBSERVATIONS USING ELECTRON BEAM FABRICATED TIPS}

The electron beam fabricated tips used in the following MFM experiments are typically $1.2 \mu \mathrm{m}$ long carbon needles with a diameter of about $200 \mathrm{~nm}$ grown on bent tungsten cantilevers and coated with a $\mathrm{Co}_{80} \mathrm{Ni}_{20}$ alloy with a nominal thickness of $20 \mathrm{~nm}$. The tips are magnetized prior to the measurements along their axis in a field of about $0.8 \mathrm{~T}$ to ensure a well defined magnetization state. All MFM observations obtained on various magnetic materials reveal a monopolelike behavior of the tips. An example is given in Fig. 2, where a typical scan line across a magneto optically written bit structure in a perpendicular magnetized $\mathrm{Co} / \mathrm{Pt}$ multilayer is shown. The similarity to the theoretically predicted MFM signal (force gradient) assuming a single polelike magnetic state of the tip scanning across a narrow domain in a perpendicular magnetized media ${ }^{8}$ is clear. Strong asymmetric peaks on either side of the bit are visible as predicted by theory. The asymmetry in the MFM signal with respect to attractive (white) and repulsive (black) forces is related to an increasing influence of van-der-Waals forces with decreasing tip-sample distance, ${ }^{9}$ a problem difficult to overcome if working in an attractive gradient at small distances in order to achieve high resolution. The observed
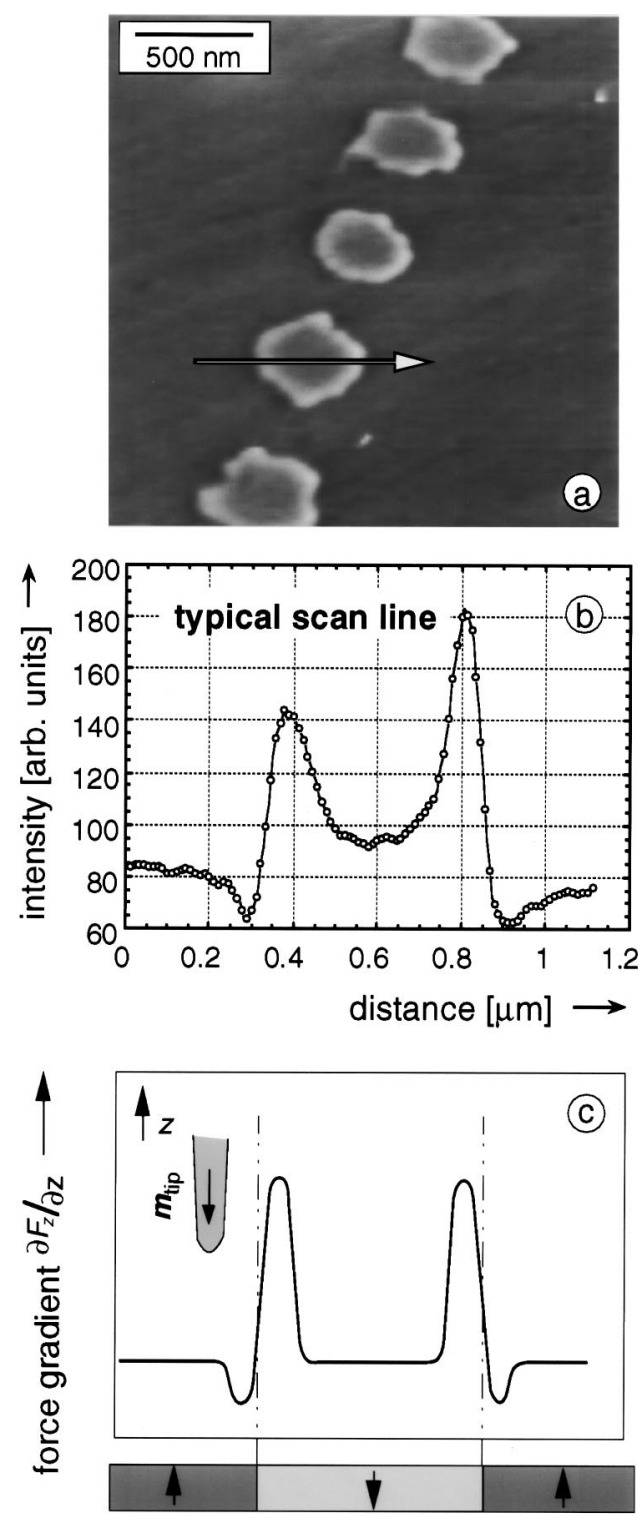

FIG. 2. (a) MFM observation of a magneto optically written bit track in a $\mathrm{Co} / \mathrm{Pt}$ multilayer. The diameter of the individual bits is about $0.5 \mu \mathrm{m}$. (b) Typical scan lines measured with the MFM reveal the theoretically derived behavior (c) assuming a monopolelike tip configuration.

asymmetry in the signal of the two transitions might be due to a misalignment of the tip with respect to the normal to the surface of the sample.

From the switching behavior of the tips observed occasionally during scanning we concluded already ${ }^{7}$ that at least the active magnetic volume of tips is in the form of a single domain element, assuming a preferred magnetization direction along the tip axis due to the shape anisotropy of the high aspect ratio thin film element. The typical switching behavior is indicated in Fig. 3. While scanning above a written bit pattern in an ME-tape the magnetic state of the tip is inverted leading to an inversion of the measured MFM signal represented by two typical scan lines before (scan 1) and after (scan 2) switching. Another reversal flips the tip magnetization back to the previous magnetic state leading again to the initially observed MFM signal (scan 3). This behavior sug- 

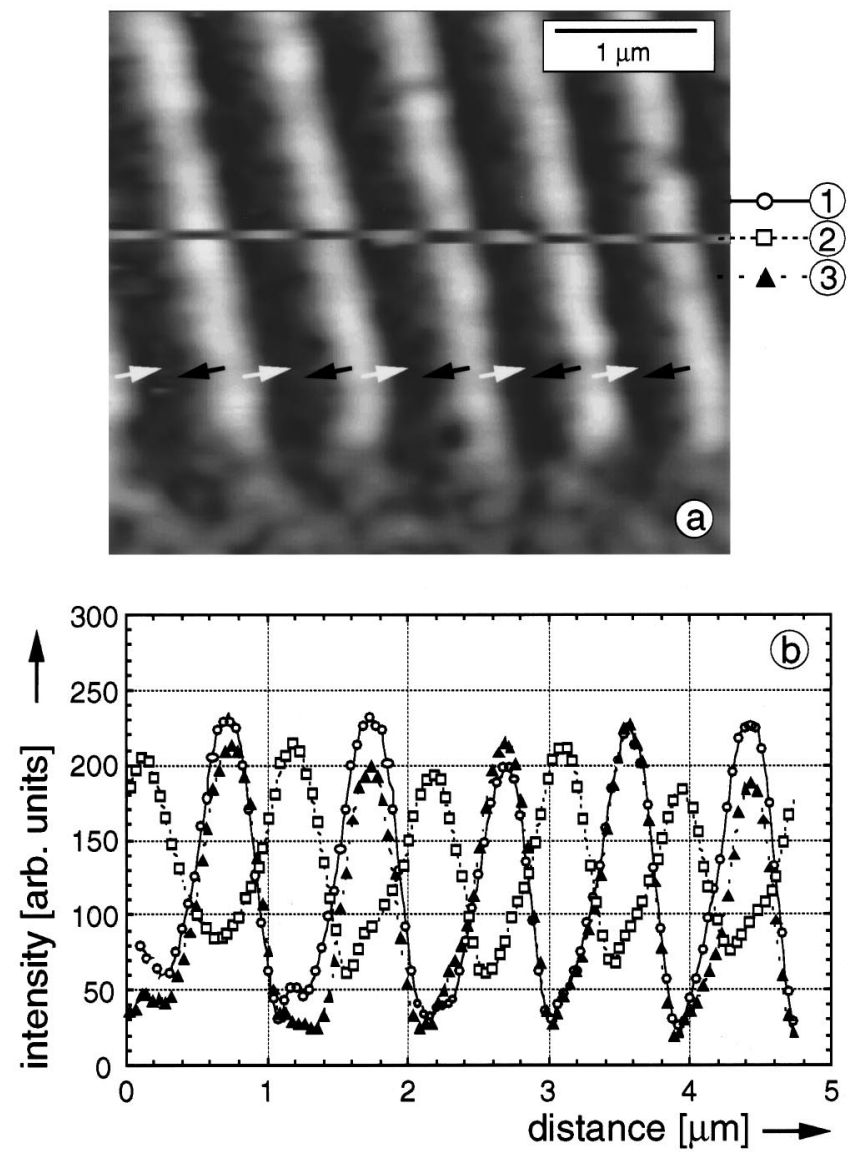

FIG. 3. (a) Reversal of the tip magnetization during scanning above a track written with a wavelength $\lambda=1 \mu \mathrm{m}$ in a CoNi ME tape. (b) The MFM signal is represented by three typical scan lines $(1-3)$ before and after the switching.

gests a square hysteresis loop for the tip magnetization, typical for single domain switching.

\section{TRANSMISSION ELECTRON MICROSCOPY OBSERVATIONS}

Various modes of transmission electron microscopy (TEM) were used to study electron beam fabricated tips in more detail. Whilst their microstructure was investigated with conventional TEM several Lorentz modes were used for their magnetic characterization. The generation of the magnetic contrast in Lorentz microscopy can be understood classically in terms of the Lorentz force leading to a deflection of the electron beam when passing through a field of magnetic induction. Only components of the magnetic flux density perpendicular to the beam are effective and the resulting deflection is linearly proportional to this flux density component integrated over the whole electron trajectory. In this way both the internal induction distribution as well as stray fields emanating from the tips are accessible, the former depending on the transparency of the tip itself for electrons of the energy used. Sample preparation, usually rather difficult in TEM, is straightforward for the electron beam prepared tips. To mount the tips in the TEM, a series of needles with different aspect ratios were grown on a tungsten wire (diameter: $10 \mu \mathrm{m})$ fixed on a standard copper grid and coated after-

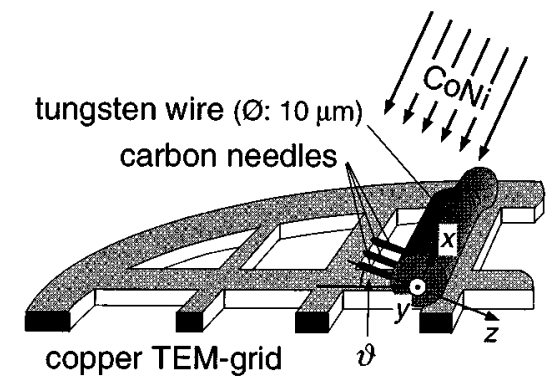

FIG. 4. Sample geometry for TEM observations: series of carbon needles are grown on top of a tungsten wire fixed on a standard copper TEM grid (with $\vartheta \approx 10^{\circ}$ ) and covered afterwards from the side with $20 \mathrm{~nm}$ of CoNi.

wards with the magnetic layer $\left(\mathrm{Co}_{80} \mathrm{Ni}_{20}\right.$ alloy with a nominal thickness of $20 \mathrm{~nm}$ ). The growth direction was perpendicular to the wire and about $10^{\circ}$ out of the grid plane (Fig. 4). In all TEM experiments described in the following, the observation direction is chosen to coincide with the evaporation direction of the magnetic material (along the $x$ axis in Fig. 4). To exclude the possibility of artefacts during observation due to electrostatic charging, additional needles were grown afterwards but remained uncoated (nonmagnetic) for comparison.

\section{A. Bright field images}

Figure 5 shows bright field images taken in a conventional TEM (CTEM JEOL 2000 FX, $200 \mathrm{keV}$ ) from Fig. 5(a) an uncovered carbon tip and from Fig. 5(b) a tip coated with
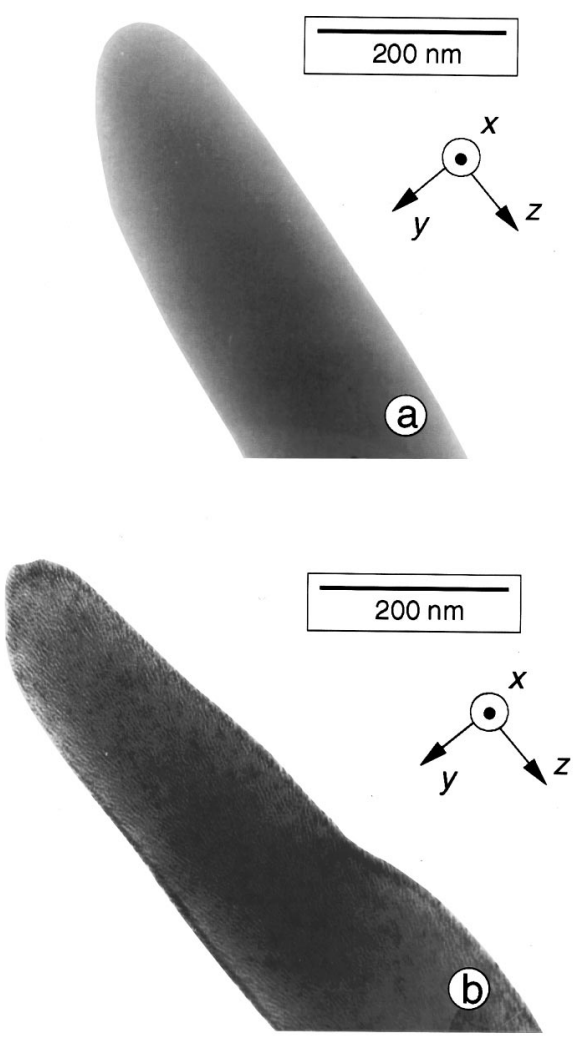

FIG. 5. Bright field TEM image of (a) an uncoated carbon needle and (b) of a carbon needle after evaporation of $20 \mathrm{~nm}$ CoNi. 

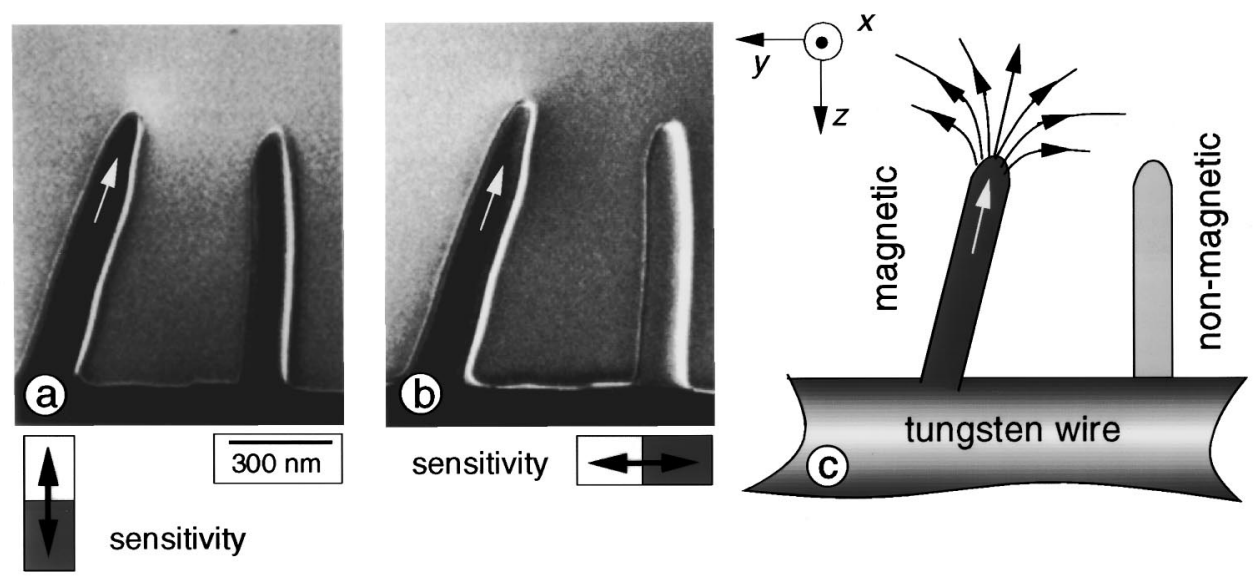

FIG. 6. (a), (b) Orthogonal Foucault images of the stray field emanating from a carbon needle covered with CoNi together with (c) a schematic interpretation. An uncovered, e.g., nonmagnetic needle is shown for comparison.

$20 \mathrm{~nm} \mathrm{Co}{ }_{80} \mathrm{Ni}_{20}$. No indication for a grain structure was found in the carbon contamination needle and electron diffraction patterns (not shown) of the uncoated tip reveal an amorphous structure. Due to their very smooth surface they form an ideal substrate for the subsequent evaporation of the magnetic material and allow the deposition of high quality films. This can be demonstrated by TEM observations of coated needles [Fig. 5(b)] showing homogenous films with a fine crystalline structure and very few defects. The latter forms a major advantage for the use in MFM as defects play an important role during the remagnetization process in magnetic thin films. The estimated grain size is about $5 \mathrm{~nm}$ and diffraction patterns obtained from continuous films prepared simultaneously on standard amorphous carbon TEM grids reveal a hexagonal structure of the $\mathrm{Co}_{80} \mathrm{Ni}_{20}$ alloy.

\section{B. Foucault mode}

Using the Foucault mode in a TEM (CTEM JEOL 2000 FX, $200 \mathrm{keV}$ equipped with a nonimmersion objective lens providing a magnetic field free space in the region of the specimen $)^{10}$ the stray field emanating from the needles can be observed. ${ }^{2,4}$ The Foucault mode relies on a slight off centering of the objective aperture obstructing half of the diffraction plane. The method shows regular magnetic contrast which is easy to interpret, but the details depend critically on the exact position and nature of the diaphragm edge which are in general not known. By changing the aperture position different components of the induction orthogonal to the electron beam can be mapped. Therefore, despite its nonlinear contrast formation, the Foucault method is well suited for many investigations and with care the direction of induction can be determined even semiquantitatively. ${ }^{11}$ We used this method to get first insights about the distribution of the stray field emerging from the tips. Results are shown in Fig. 6 where in Fig. 6(a) the sensitivity axis of the magnetic contrast is oriented near parallel to the tip axis whereas in Fig. 6(b) it is near perpendicular. A nonmagnetic needle is shown for comparison. By combining information from both orientations, the stray field distribution, shown schematically in Fig. 6(c), is inferred. The monopolelike behavior of the tip, already assumed from the interpretation of MFM images obtained with this type of needles, is apparent. No stray field is emanating from the parallel sidewalls of the needles clearly demonstrating the advantage of the electron beam fabrication method producing amorphous tiny cylindrical carbon needles with a rounded apex and very smooth surface. The magnetization configuration was stable up to the maximum field of $10 \mathrm{mT}$ provided by a magnetization stage within the TEM. Reversing the tip magnetization in an external field of $0.4 \mathrm{~T}$ led to inverted contrast, further confirmation of the magnetic origin of the observed contrast.

\section{Differential phase contrast (DPC) mode}

With the DPC mode implemented in a scanning TEM (STEM VG HB5, $100 \mathrm{kV}$ ) it is possible to map quantitatively two orthogonal in plane components of the magnetic induction perpendicular to the electron beam on line by using a quadrant detector. ${ }^{12}$ In the following, DPC imaging is used to get first insights into the mechanism involved during the remagnetization process of electron beam fabricated tips. From the tip switching behavior observed occasionally in MFM experiments (see above) we concluded already that at least the active volume of the tip should only consist of a single domain revealing a square hysteresis loop. In order to check the validity of this inference, we performed magnetizing experiments within the electron microscope along with observations of the magnetic state of the tip. As the tips are near opaque for the maximum electron energy in this microscope it was only possible to image the emanating stray field. Moreover, only remanent states could be observed as the field was supplied by pulsing the objective lens which is not excited when imaging the magnetic induction. Starting from a state saturated along the tip axis ( $-z$ direction) we applied increasing field pulses with a component $H_{\text {ext }}$ along the $+z$ direction in order to remagnetize the tip. The results are summarized in Fig. 7 where the two simultaneously mapped orthogonal induction components are shown together with schematics of the stray field distribution inferred from the observed contrasts. A magnetic and a nonmagnetic needle are again shown for comparison. The initial remanent state after 

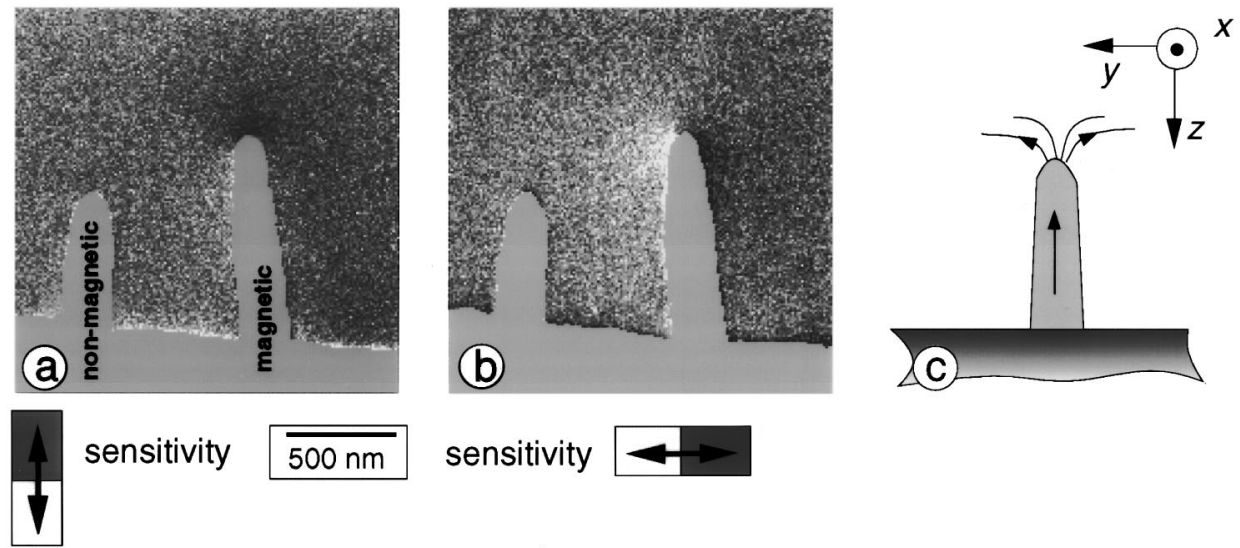

sensitivity
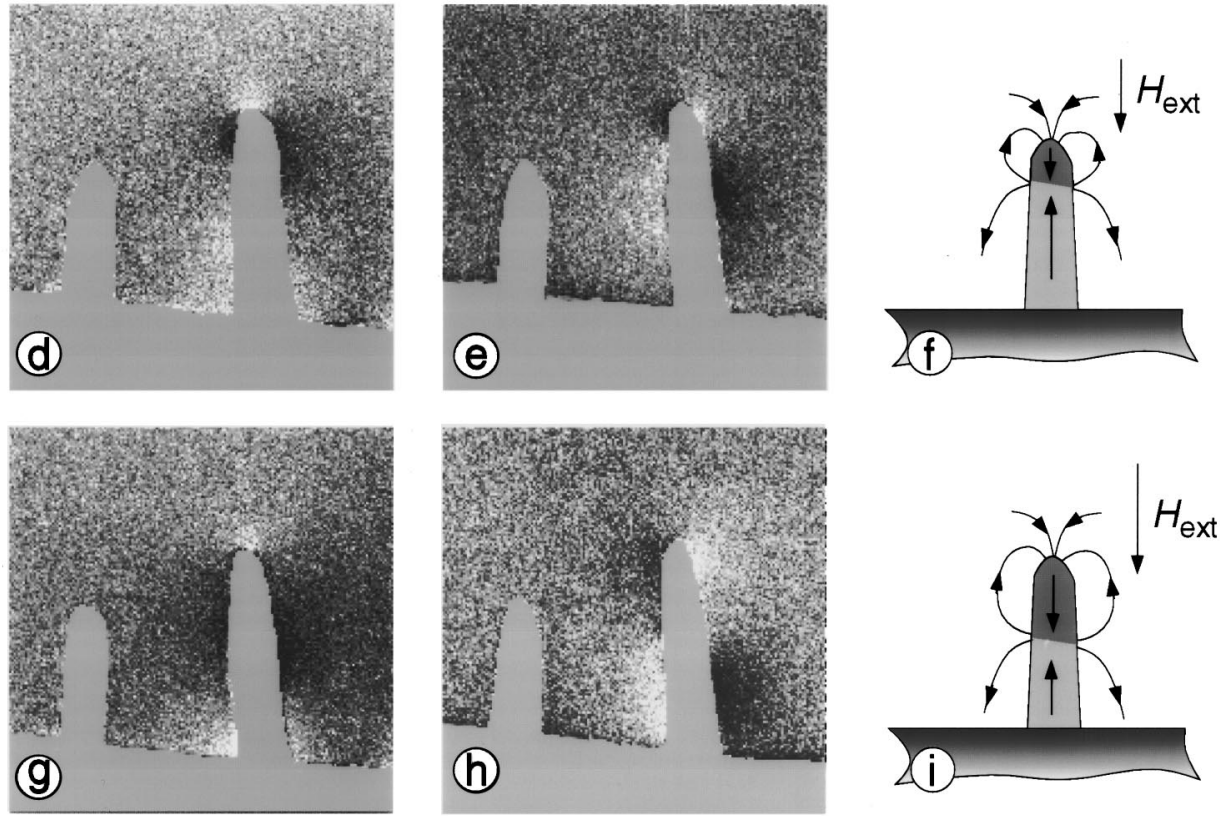

FIG. 7. Switching behavior of the tips in an external field supplied by the microscope objective lens. DPC images of different remanent states are shown together with a schematics of a possible domain configuration within the tip.

saturation along the tip axis [Figs. $7(\mathrm{a})$ and $7(\mathrm{~b})]$ reveals the monopolelike contrast already observed in the Foucault experiments. Only a weak stray field is emanating from the immediate apex region and no stray field is observed along the parallel side-walls of the needle. Below lens field pulses of about $0.2 \mathrm{~T}$ the initially observed contrast remained unchanged. After applying a higher field pulse the contrast at the tip end is inverted and additional stray field is seen emanating from the side walls [Figs. 7(d) and 7(e)]. A hypothetical magnetization distribution consistent with the observed contrast is shown schematically in Fig. 7(f) involving two domains magnetized along the tip axis separated by a head-on wall [note: such highly charged walls, known from the easy axis magnetization process in thin films, tend to fold ("zigzag" wall) in order to reduce the attended stray field energy]. The inferred picture coincides with the observation, that the observed contrast can be shifted continuously along the tip axis with slightly higher field pulses [Figs. 7(g) and 7(h)]. This might be attributed to a movement of the head-on wall [Fig. 7(i)]. No indication was found in the experiments that the immediate end of the tip consists of more than one domain magnetized along the tip axis, consistent with the MFM experiments. Moreover the reduced signal in the reversed MFM scan [scan 2 in Fig. 3(b)] might be understood in such a way that the $+z$ component of the tip magnetization in the intermediate state [Fig. 7(d)-7(i)] is partially cancelled by the domain still magnetized along the $-z$ direction.

\section{Coherent Foucault (CF) mode}

Using the coherent Foucault (CF) imaging technique ${ }^{13}$ in a Philips CM 20 CTEM/STEM (200 kV) equipped with a thermally assisted field emission gun (FEG) providing high spatial coherence, the magnetic structure can be imaged directly in the form of magnetic interferograms. In this way immediate quantitative access to the magnetic induction distribution within the samples is provided, impossible in the conventional Foucault technique as mentioned above. Depending on the position and nature of the aperture, different components of the magnetic induction can be imaged as in the conventional Foucault mode but additional quantitative information can be obtained from the interference pattern, a 

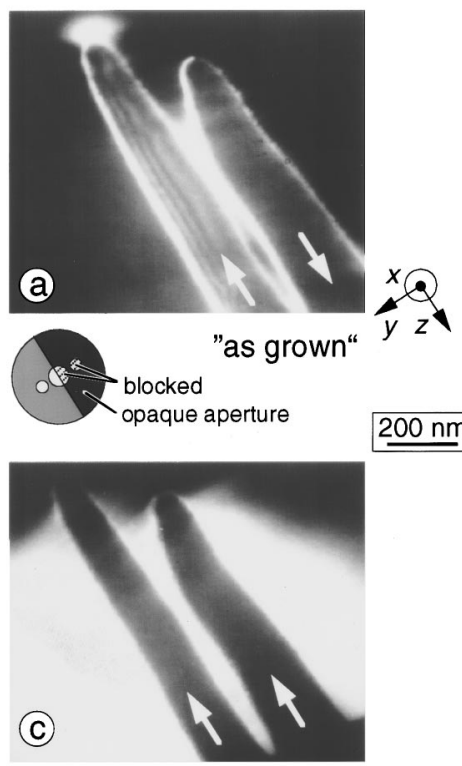

(C)

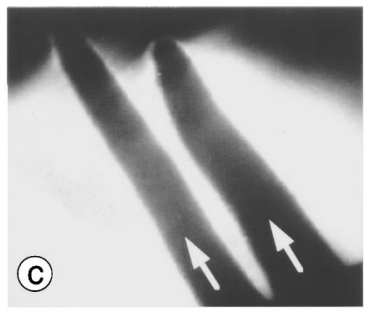

$200 \mathrm{~nm}$

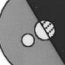

$\therefore$
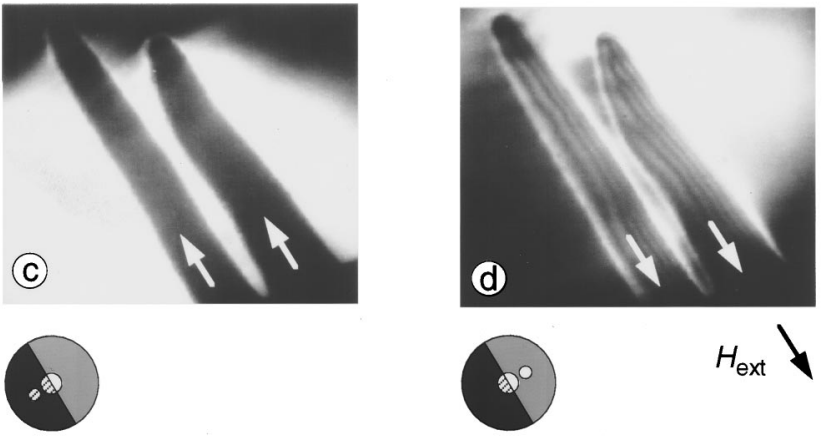

FIG. 8. Coherent Foucault images of different tip remanent magnetization states. The central portion of the diffraction patterns together with the position of the opaque aperture used is shown schematically in the insets.

kind of in-line hologram. For the theoretical description of this imaging mode a quantum mechanical treatment of the electron-specimen interaction is required. A detailed analysis together with two-dimensional computer simulations for various magnetization distributions can be found elsewhere. ${ }^{14}$ However, for the understanding of the experiments described in the following it is sufficient to mention the basic result: if the interaction is purely magnetic, the interferogram can be interpreted in terms of magnetic lines of force and thus reveals immediately the variation of the magnetic induction within the sample. We used this technique to investigate the domain structure of the MFM thin film tips with different remanent magnetization states. CF images are shown in Fig. 8 for two needles grown next to each other; different images reveal the as-grown state as well as after application of different fields along the tip axis. The field is provided by tilting the sample through $90^{\circ}$ around the $y$ axis and exciting the objective lens to its maximum value of $0.7 \mathrm{~T}$ (note that the objective lens is usually not excited when imaging magnetic structures, instead additional, so called Lorentz lenses are used to provide field-free imaging of the specimen). In the as-grown state [Fig. 8(a)] the magnetization of the two needles is antiparallel. A schematic of the central portion of the diffraction pattern obtained from this state is shown in the inset. Adjusting the position of the aperture enables domains lying along the $-z$ direction to contribute to the signal. Only in this region are fringes visible, whereas electrons passing through areas magnetized along the $+z$ direction are blocked leading to homogeneous dark areas. After saturation of the sample in the $-z$ direction fringes become visible in both needles [Fig. 8(b)] without changing the position of the aperture in the diffraction plane.
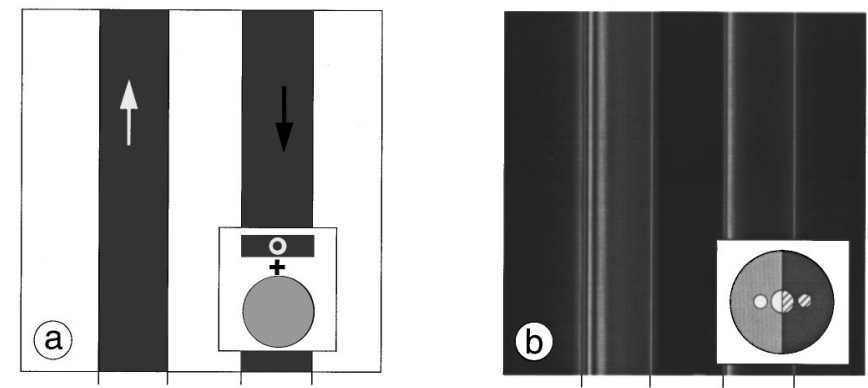

FIG. 9. Computer simulations of the experiment in Fig. 8(a). The sample (a) consists of two carbon cylinders covered with a magnetic thin film magnetized homogeneously and antiparallel. The interferogram (b) is calculated for a semi-infinite opaque aperture cutting just the central spot of the diffraction pattern.

After inverting the sensitivity axis by adjusting the opaque aperture in the back focal plane of the objective the fringes disappear in both needles [Fig. 8(c)]. Saturation of the sample in the $+z$ direction causes the fringes again to appear in both needles [Fig. 8(d)] clearly demonstrating their magnetic origin. Note that the separation of the fringes varies across the needle diameter and that this variation is inverted from Fig. 8(b) to 8(d). This can be understood in the quantum mechanical description of the image formation if an additional phase shift is included arising from the varying sample thickness across the diameter of the cylindrical needles. The experimental observations can be confirmed by computer simulations of the interferograms. Results are given in Fig. 9 for two carbon wires covered with a magnetic thin film magnetized antiparallel and homogeneously along the wire axis. A schematic cross section is indicated in the inset of Fig. 9(a). The interferogram derived out of this configuration is shown in Fig. 9(b) if a semi-infinite opaque aperture is inserted in the diffraction pattern to block just the central spot. The resemblance with the observations in Fig. 8(a) is apparent.

\section{CONCLUSIONS}

By imaging the stray field of electron beam fabricated MFM tips with the conventional Foucault and the differential phase contrast mode in transmission electron microscopy it was possible to get first direct insights into their magnetic behavior. The observations confirmed the basic features already claimed indirectly out of MFM observations using these tips. The emanating stray field is concentrated at the immediate apex region of the tiny needles emphasizing their high-resolution capability if used in MFM. Although stray field observations are only an indirect method with respect to the domain structure within the tip, no indication could be observed during the remagnetization process that the immediate apex region consists of more than one domain magnetized along the tip axis. Unfortunately, the precise orientation of the needles relative to the applied lens field is not exactly known due to uncertainties in the sample geometry (bending of the needles during the evaporation of the magnetic thin film). Therefore a comparison of the switching field value found in the experiments with the upper limit calculated 
from Stoner-Wolfarth theory is not significant at this stage. Moreover, the observed switching mechanism with the assumed generation of a head-on-wall already forms a deviation from the theory of single domain switching assuming only coherent rotation. Future experiments are necessary to identify the typical remagnetization process within the tips on samples with accurately known geometry. Due to the high electron transparency of the coated carbon needles it is possible to observe the domain structure of the tips directly. This could be demonstrated in preliminary magnetizing experiments using the coherent Foucault imaging technique to map the flux distribution within the magnetic layer itself. No indication of reverse domains could be observed in needles at remanent magnetization states and the magnetization was found to lie always homogeneously along the tip axis. This forms a first direct proof for a single domain and therefore well defined magnetic state of electron beam fabricated tips-an important result regarding their capability for improved MFM.

\section{ACKNOWLEDGMENTS}

The authors would like to thank Dr. D. M. Donnet and L. Zhou for practical help. We are grateful to the CAMST project of the European Community for financial support.
M.R. further acknowledges financial support from the European Commission within a HCM research fellowship.

${ }^{1}$ P. Grütter, H. J. Mamin, and D. Rugar, in Scanning Tunneling Microscopy II, Springer Series in Surface Science, edited by R. Wiesendanger and $\mathrm{H}$. J. Güntherodt (Springer, Berlin, 1992), Vol. 28, p. 151.

${ }^{2}$ S. McVitie and U. Hartmann, 49th Annual Meeting of the Electron Microscopy Society of America (San Francisco Press, San Francisco, CA, 1991), p. 770.

${ }^{3}$ P. Grütter, D. Rugar, H. J. Mamin, G. Castillo, C.-J. Lin, I. R. McFadyen, R. M. Valetta, O. Wolter, T. Bayer, and J. Greschner, J. Appl. Phys. 69, 5883 (1991).

${ }^{4}$ K. Sueoka, F. Sai, K. Parker, and T. Arnoldussen, J. Vac. Sci. Technol. B 12, 1618 (1994).

${ }^{5}$ G. Matteuchi, M. Muccini, and U. Hartmann, Appl. Phys. Lett. 62, 1839 (1993).

${ }^{6}$ C. Schönenberger and S. F. Alvarado, Z. Phys. B 80, 373 (1990).

${ }^{7}$ M. Rührig, S. Porthun, and J. C. Lodder, Rev. Sci. Instrum. 65, 3224 (1994).

${ }^{8}$ D. W. Abraham and F. A. McDonald, Appl. Phys. Lett. 56, 1182 (1990).

${ }^{9}$ J. J. Sáenz, N. Garcia, and J. C. Slonczewski, Appl. Phys. Lett. 53, 1449 (1988).

${ }^{10}$ J. N. Chapman, J. Phys. D: Appl. Phys. 17, 623 (1984).

${ }^{11}$ S. J. Hefferman, J. N. Chapman, and S. McVitie, J. Magn. Magn. Mater. 83, 223 (1990).

${ }^{12}$ J. N. Chapman, I. R. McFadyen, and S. McVitie, IEEE Trans. Magn. MAG-26, 1506 (1990).

${ }^{13}$ J. N. Chapman, A. B. Johnston, L. J. Heyderman, S. McVitie, W. A. P. Nicholson, and B. Bormans, IEEE Trans. Magn. 30, 4479 (1994).

${ }^{14}$ A. B. Johnston and J. N. Chapman, J. Microsc. (in press).

Published without author corrections 